\title{
Fracture Healing in a Denervation and/or Nerve Ending Interpositioning Model in the Rat
}

\author{
Cagri Yegengil ${ }^{1}$, Mahmut Pekedis ${ }^{2}$, Hasan Yildiz $^{2}$ \\ ${ }^{1}$ Bozyaka Training and Research Hospital, Orthopedics and Traumatology Clinic, Izmir, Turkey; ${ }^{2}$ Ege University, Engineering Fac- \\ ulty, Mechanical Engineering Department, Izmir, Turkey. \\ Email:mahmut.pekedis@ege.edu.tr
}

Received April 14 $4^{\text {th }}, 2011$; revised May 17 ${ }^{\text {th }}, 2011$; accepted July $3^{\text {rd }}, 2011$.

\begin{abstract}
Background: In this experimental study, we aimed to determine the possible changes in fracture healing due to denervation and/or nerve ending interpositioning. Methods: 50 Wistar Albino type male rats were divided into three study groups. A standard transverse diaphysial fracture in the femurs of the same side of all subjects under anesthesia was created and the fracture was fixed intramedullarily. While preserving the structural integrity of the sciatic nerve in the first group, neurectomy to the nerve in the second group was performed. In the third group, following the sciatic nerve cut, the proximal end of the nerve were interposed the fracture line. After a 28-day observational period, the callus formation in the subjects was examined radiologically, biomechanically and histopathologically. Results: Among all groups, the third group subjects showed significant increase in radiological area measurements when they are compared to the second group rats. There was no significant difference in biomechanical measurements of fractured femurs of the three groups. In histopathological evaluations, it was observed that denervation had increased the thickness of the cartilage and the number of the chondrocytes and osteoclasts significantly but decreased the number of fibroblasts compared to the control group. In addition to the denervation nerve ending interpositioning increased the bone thickness and the number of the osteoblasts but decreased the number of the osteoclasts significantly. Conclusions: While radiological observations exhibit that nerve ending interpositioning has resulted more hypertrophic callus formation, histopathological evaluations led us to that denervation created partial (immature) callus formation and nerve ending interpositioning demonstrated larger but immature callus formation.
\end{abstract}

Keywords: Denervation, Nerve Ending Interposition, Fracture Healing, Callus Formation

\section{Introduction}

Fracture healing is defined as a complex problem that includes coordination of different processes in the literature [1]. Existence of neural formation in bone tissue was first documented in 1545 [2]. Neural control of new bone formation hypothesis extends to $1930 \mathrm{~s}$ [3]. The neural tissue was thought only in periost, therefore neural tissue of bone is generally disregarded. Sudeck atrophia related to chronic regional pain syndrome, heterotypical ossification that is observed in patients with head trauma and Charcot diabetic neuroarthropaty point out that an interaction between nervous system and muscle-skeleton system should exist [2]. Rapid joining with plenty of callus formation in patients with neurological damage such as head trauma, traumatic paraplegia etc. and reduced ossification in some other neurological pathologies such as Charcot disease were observed. Long bones have a rich innervation by myelinated and non myelinated nerve fibers. Functions and endings of these nerve fibers were still not known, while blood flow regulative roles of sympathetic nerves are known [4].

Denervation is one of the factors that effect fracture healing. Concordantly, experimental studies related to effects of denervation on fracture healing process exist in the literature. One of these several denervation models that were used in these studies is neurectomy. Existence of neurological tissue and its effect on callus were explained by methods devoted to immunohistochemical analysis of some neuromarkers such as growth-associated protein 43 (GAP-43) and protein gene product 9.5 (PGP-9.5) that points existence of nerve tissue in examined fractural callus by forming denervation. A reinnervation without denervation after fracture which was formed experimentally was given by Li et al. [5]. Despite the existence of sympathetic and sensorial nerve 
fibers in the periost and bone; and their specific receptors, effect mechanism to callus formation and maturation is not clear [4].

\section{Materials and Methods}

\subsection{Study Groups}

In the present study, 45 Wistar-Albino rats that were 12 - 14 weeks old with average weight of 299 grams were used. Ethical Board approval was obtained from Ege University Animal Ethics Board for this study. 45 rats were divided into three groups. Standard transverse fractures were created in right femur diaphyse that were determined intramedullary for all groups. As difference, in the first group integrity of nervous sciatica was preserved. 15 millimeters of resection was applied to nervous sciatica in the second group. In the third group, the proximal part of the nerve was stabilized to fracture line from free nerve end by applying sharp injury model with a complete transvers cut to nervous sciatica in fracture line level, while the distal part of the nerve was excised widely (Figure 1).

\subsection{Surgical Procedure}

Anesthesia was applied to test subjects by administrating Ketalar (Eczacıbaşı Warner Lambert) which includes $100 \mathrm{mg}$ Ketamine Hydrochloride active substance as $100 \mathrm{mg} / \mathrm{kg}$ and Xylazine (Rompun Bayer İstanbul) as 10 $\mathrm{mg} / \mathrm{kg}$ subcutaneously. A single dose of Ampisina 50 $\mathrm{mg} / \mathrm{kg}$ was administrated as antibiotic prophylaxis. Long axis of femur was entered by parallel skin incision from external side of right thighbone of test subjects and nervous sciatica was reached by cutting the gluteal muscle parallel to the first incision. After that, femur diaphysis was exposed by separating from adjacent muscle tissues with dissection. Kirschner wire of $1 \mathrm{~mm}$ thickness was sent from proximal of the femur intramedullary via electric drill and the upper part of the bone was cut such that $1-2 \mathrm{~mm}$ of wire came out of the bone. Transverse diaphysis fracture was formed via a bone forceps in femur diaphysis. Fascia and skin in all test subjects were closed as 2 layers; in the first study group,

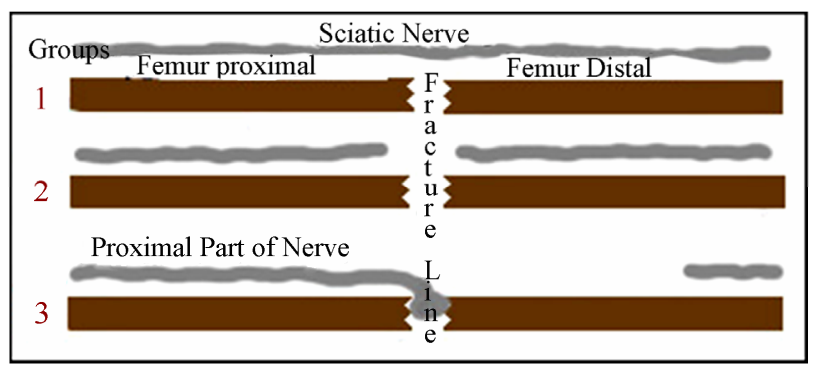

Figure 1. Study groups (nervous sciatica and femur are shown with light and dark color respectively). structural integrity of nervous sciatica was preserved. In the second group, denervation was aimed by applying 15 $\mathrm{mm}$ of resection. In the third group, sharp injury model was applied with \#15 lancets from approximately $5 \mathrm{~mm}$ distal part of the fracture line to prevent high tension on nervous sciatica. After then, upper end of the nerve was stabilized from epineureum with 10/0 nylon stitch material (Figure 2). Lower part of the nerve was excised at the level as the lowest level as possible.

Test subjects were allowed to move freely in wire cages. Operated extremities of subjects were followed in terms of sufficient stabilization and infection. It was observed that test subjects used the other three extremities and protected the operated extremity as expected. After four weeks, all test subjects were sacrified by administrating high dose of Pentotal (Pantobarbutal 50 $\mathrm{mg} / \mathrm{kg}$ ) intraperitoneally. Both femurs of all test subjects were removed by protecting the soft tissues at $1 / 3$ middle part of femur diaphysis. Kirschner wires of all test subjects were removed.

\subsection{Statistical Evaluation}

The statistical analysis of the results was done on "SPSS 15.0 " statistics package program with a precision of 95\%. During the statistic analysis of data, Wilcoxon Signed Ranks, Mann-Whitney 'U', Spearman's correlation methods were used.

\subsection{Radiological Evaluation}

A-P direct pictures of all femur samples were taken on the day of scarification. Imaging process was performed with high resolution digital radiography system. The obtained images were evaluated in "Image Viewer R 10.2" software of PACS product of Philips Medical Systems company as C: 8600 (contract), W: 9440 (brightness) with their standard values digitally. During the evulation of images, methaphisodiaphiser segment areas of the

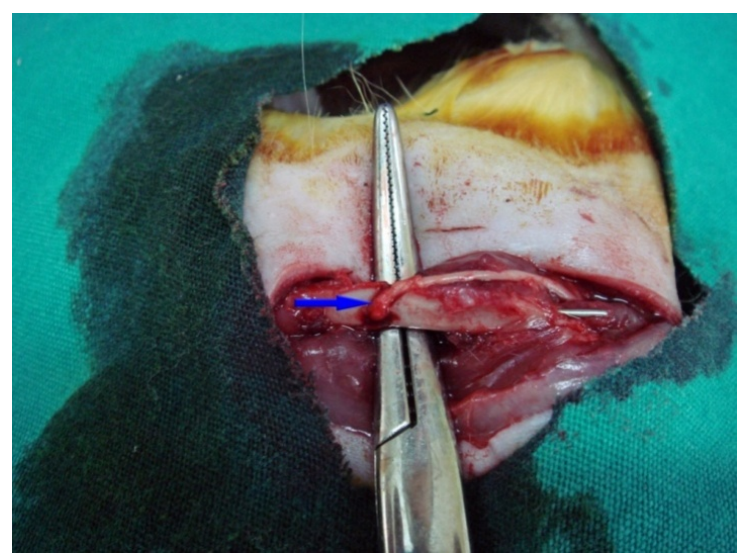

Figure 2. Interposition of proximal end of nervous sciatica to the fracture line (arrow). 
right femurs that callus formation was observed and the left femurs that callus formation was not observed were calculated. To determine the isolated diaphysis area of the femur, vertical lines were drawn from trochanter minor level in upper end of femur and from joining level of femur condyles with diaphysis to the longitudinal axis of the femur and the area between these lines were calculated. With this technique, area increase that callus tissue made to the diaphysis was observed (Figure 3).

\subsection{Histopathological Evaluation}

After the direct graphs were taken, the right femurs of eight test subjects from the first and the third group and seven test subjects from the second group were separated for histopathological evaluation. Because two of the samples from the first group were damaged during the transportation, the histopathological evaluation of this group was performed on six samples. Numbered samples were fixed in the room temperature with $10 \%$ formal solution. Following this process they were decalcified in the room temperature with $10 \%$ formic acid for a week, with $48-72$ hours intervals. After the decalcification, 2 - $3 \mathrm{~mm}$ tissue sections were taken and blocked into the paraffin. $6 \mathrm{~mm}$ longitudinal and transversal sections were taken via a microtome and coloration was performed with HE (haematoxylon-eosin) and alkaline blue. After this process, 6 to 8 sections were examined in light microscope with $20 \times, 40 \times, 100 \times$ and $400 \times$. Cell count in per unit area from the most cellular regions of samples (osteocyte, osteoblast, osteoclast, fibroblast, chondrocyte) and bone and cartilage measurement of the callus were performed (Figure 4). Obtained data were evaluated for the groups with Mann-Whitney U test.

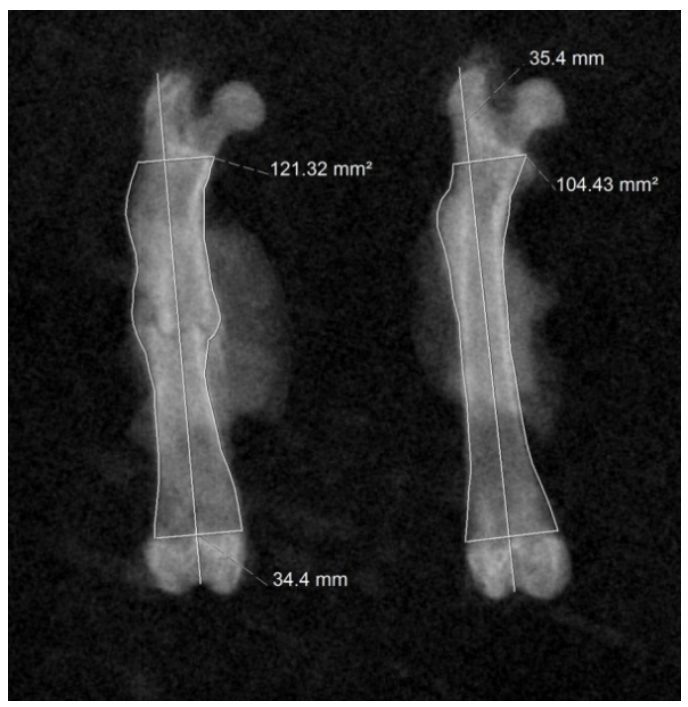

Figure 3. Isolated femur diaphysis areas of the number 16 number test subject of group 2.

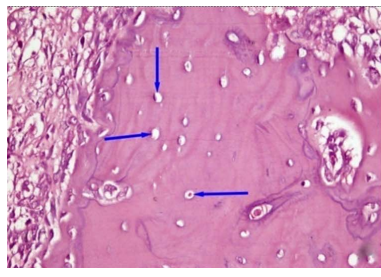

(a)

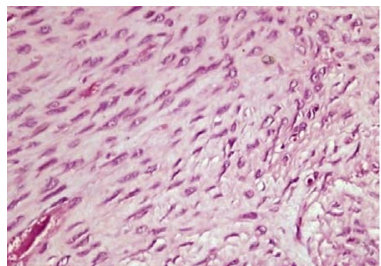

(c)

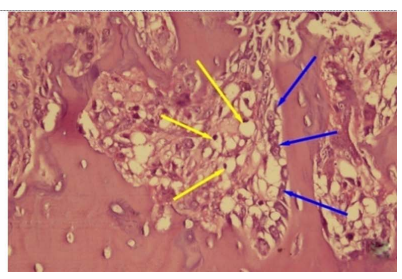

(b)

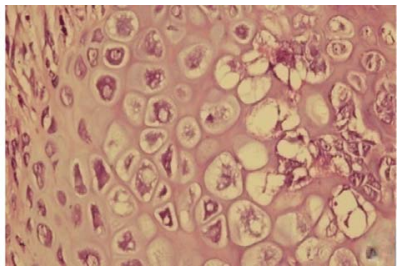

(d)
Figure 4. Bone cells in the unit area (a) osteocytes (blue arrows); (b) osteoblasts and osteoclasts (blue arrows show osteoblast cell alignment, yellow arrows show multinuclear osteclasts); (c) fibroblasts; (d) chondrocytes.

\subsection{Biomechanical Evaluation}

The right femurs where callus formation was observed and opposite side healthy femurs of eight samples from the first and the second group; seven samples from the third group were separated for biomechanical evaluation. Then, all femurs were embedded in to bone cement in vertical direction via a goniometric ruler. In the study, SHIMADZU Autograph AG-I 10kN was used as loading device. The axial load is applied to the specimens at a speed of 1 millimeter per minute $(1 \mathrm{~mm} / \mathrm{min})$. The result of measurements was recorded to a computer as force $(\mathrm{mN}) /$ displacement $(\mathrm{mm})$ data. Their hardness, elasticity and energy storing capacities of specimens were computed by using force-displecement diagrams.

\section{Results}

\subsection{Radiological Evaluation}

In the right femurs of all groups, callus tissue formation was observed at the end of the fourth week. The bridging of fracture openings in all groups were observed radiologically as periosteal bridging shaped (external callus). While there was no meaningful difference between the first and the second groups, and the first and the third groups during the comparison of isolated diaphysis fields in the right femurs which showed callus formation, there was a statistically meaningful difference between the second and the third groups (Figure 5).

\subsection{Biomechanical Evaluation}

There was no statistically meaningful difference between comparisons of fractured bones of three study 


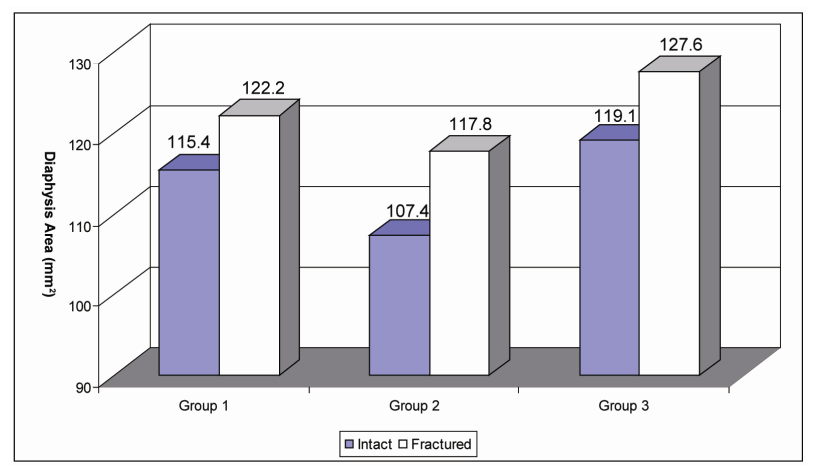

Figure 5. Average areas of isolated femur diaphysis.

groups in terms of studied parameters: elasticity, hardness and energy storing ability.

\subsection{Histopathological Evaluation}

While callus formation of different degrees and phases was observed in all of the study groups, generally smaller and more developed stage of callus formation was observed in the first group (Figure 6), and more hypertrophic and earlier staged callus formation was observed in the second and the third groups.

In the group comparisons of callus bone and cartilage parameters (osteocyte, osteoblast, osteoclast, fibroblast, chondrocyte) with the method of cell count per millimeter square, it was observed that denervation increased the cartilage thickness, the chondrocyte number and the osteoclast number considerably (pct $=0.033$, $\mathrm{pcn}=$ 0.018 , pon $=0.024$ respectively), and decreased the number of fibroblast ( $p=0.042$ ) depending on the control group. In the denerved group, there was also an increase in the number of osteocyte and bone thickness, even though it was not considerably so $(11.06 \%$ and $26.25 \%$ respectively). It was also observed that the nerve ending interposition in the fractured callus considerably increased the bone thickness compared to the first group,

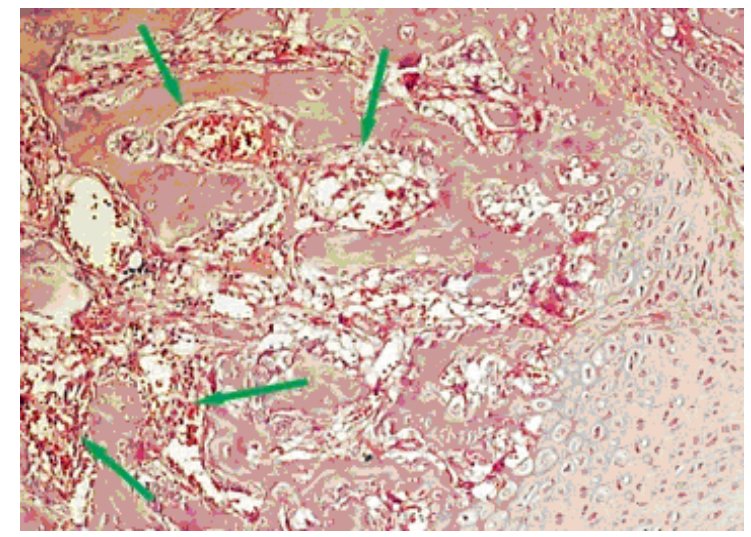

Figure 6. Apparent trabecular bone formation regions in group 1 (arrows).

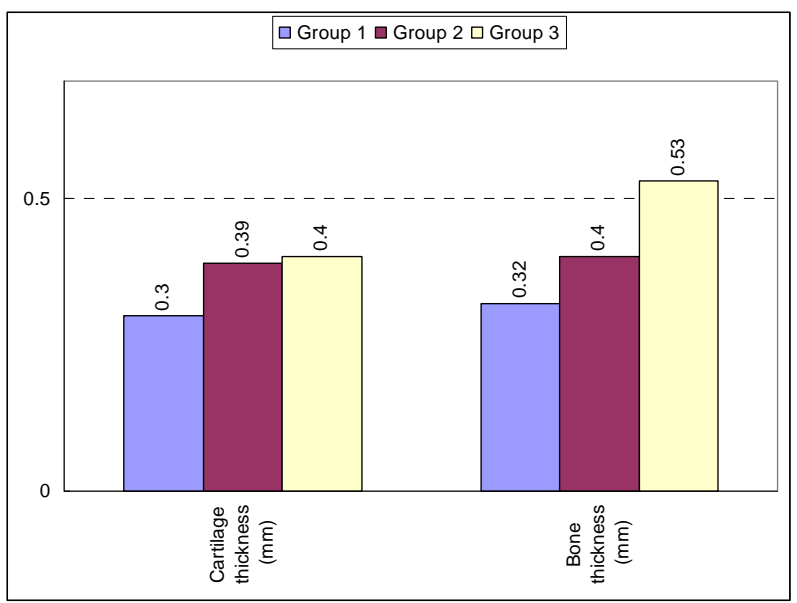

Figure 7. The bone-cement thickness comparisons.

and increased the number of osteoblast compared to the first and the second group ( $p=0.045, p=0.034$ and $p=$ 0.004 respectively). It also decreased the number of osteoclast compared to the second group $(p=0.016)$. Other histopathological parameters were similar to denervation group (Figures 7 and 8 ).

\section{Discussion}

First of all, the formation of fracture on femur following the nervous sciatica neurectomy leads us to believe that the model can not denerve the bone completely. Following nervous sciatica neurectomy of rats, Frymoyer and Pope and Hukkanen et al. formed fracture in fibula and tibia respectively [6,7]. On the other hand Madsen et al. created fracture in tibia after sciatic and femoral nervous neurectomy [8]. As a quantifier indicator showing how much neurectomy denerved the relative fracture, Hukkanen et al. performed neuromarker analysis on callus with immunohistochemical methods [7]. Owing to

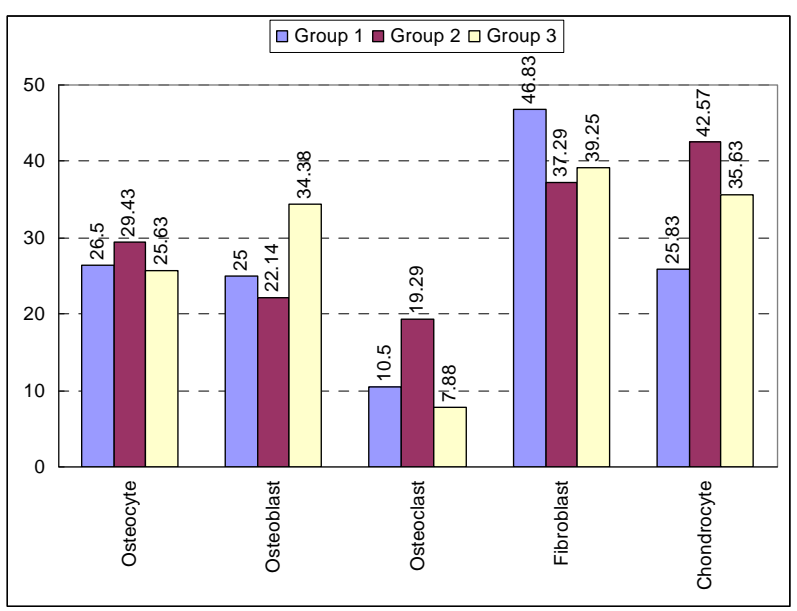

Figure 8. The number of cells comparisons. 
their findings, tibial fracture callus is not solely denerved by nervous sciatica, but it can also be innervated by other sources. For this reason, in the following study, Madsen et al. performed the neurectomy in both the nervous sciatica and femoral nerve [8]. The analysis of the callus with immunohistochemical methods in terms of neural tissue may clarify (as in more or less) the denervation amount in this study.

How do the denervation models in rats affect the healing of fracture?

When the fracture healing is evaluated in radiography and physical examination findings in clinics, quantifier evaluation may be difficult [9]. It is observed that this evaluation is done with radiographic, histopathologic, densitometric and biomechanical measurements on rat femurs $[4,6-8,10,11]$. Even though nervous sciatica denervation was bigger, flat and calcific looking, Aro et al. observed that in rat tibia fractures which are created as intramedullary lead to a formation of callus which exhibits more irregular density [4]. In the control groups, callus was oval shaped and showed more regular density distribution. Yüce et al. discovered radiologically that the fibula fractured fragments of rats which had sciatic denervation were combined on the $28^{\text {th }}$ day and while a minimal opening had found in the control group 10. In their evaluation which was made with radiological scoring system, Hukkanen et al. and Madsen et al. discovered there was primer fracture healing in normal innerve fractures, and there was secondary healing in denerve cases which is characterized by large callus formation $[7,8]$. In the first group, considering the comparisons of fractured bones of the radiological findings at denervation, no change was discovered which is similar to the literature.

During the healing of fibula fractures of fibula fractures of rats whose nervous sciatica was denerved, Frymoyer snd Pope found an increase in biomechanical properties such an elasticity, energy storing and hardness on the $15^{\text {th }}$ and $20^{\text {th }}$ days [6]. During the earlier stages of fracture healing (first 15 days), Aro et al. found that the maximum fracture force increased in denerve group 4. Hukkanen et al. and Madsen et al. found that maximum bending moment, energy storing capacity and hardness values in denerve group were somehow lessened on the $35^{\text {th }}$ day when compared to control group $[7,8]$. In this study, the fact that no meaningful difference was observed between fractured and intact bone biomechanically resulting denervation and nerve end interposition model are compatible with the literature. This may be due to the fact that biomechanical evaluation in this study is done with axial lading test as opposed to fracturing from three points test in previous studies.
The histopathological evaluation results of the denervation group, considering the stages of the fracture healing, point to the fact that denervation causes a delay in callus formation process. In the control group, a callus formation was observed and it is smaller than that of denervation and nerve ending interposition groups, but it was found to be is in more devoloped stage. In the healing of fibula fractures of rats whose nervous sciatica was denerved, Frymoyer and Pope observed histological increase [6] on the $15^{\text {th }}$ and the $20^{\text {th }}$ days (using a scoring system which consists of hematom, callus, joining and compact bone formation measurements). Yüce et al. discovered lamellar bone formation and osteoclasts on the $28^{\text {th }}$ day histologically, and on control side, an earlier stage of callus formation where the fibrosis and cartilage tissues were dominant [10]. In their study concerning the development of sensory innervations in rat tibias, Gajda et al. observed an increase in osteoclastic activity in denerved bones [12].

How does the performing of nervous sciatic denervation with nerve ending interposition in rats affect the fracture healing in femur?

No experimental models concerning the interposition of nerve end to fracture line were encountered in the literature. The fact that a more hypertrophic callus formation was observed in the nerve end interposition of this study radiologically when compared to denervation may be secondary to various physical and/or chemical stimulus in the fracture line of the nerve. Since neither immunohistochemical nor molecular analysis was done about this subject, no further comments can be made. In the histopathological evaluation, it can be observed that nerve end interposition leads to a bigger but immature callus formation when compared to denervation.

\section{REFERENCES}

[1] A. Schindeler, M. M. McDonald, P. Bokko and D. G. Little, "Bone Remodeling during Fracture Repair: The Cellular Picture," Seminars in Cell and Development Biology, Vol. 19, No. 5, 2008, pp. 459-466. doi:10.1016/j.semcdb.2008.07.004

[2] K. B. Jones, A. V. Mollano, J. A. Morcuende, R. R. Cooper and C. L. Saltzman, "Bone and Brain: A Review of Neural, Hormonal, and Musculoskeletal Connections," The Iowa Orthopaedic Journal, Vol. 24, 2004, pp. 123132.

[3] D. J. Hurrell, "The Nerve Supply of Bone," Journal of Anatomy, Vol. 72, No. Part 1, 1937, pp. 54-61.

[4] H. Aro, E. Eerola, A. J. Aho and R. Penttinen, "Healing of Experimental Fractures in the Denervated Limbs of the Rat," Clinical Orthopaedics and Related Research, Vol. 155, 1981, pp. 211-217.

[5] J. Li, T. Ahmad, M. Spetea, M. Ahmed and A. Kreicbergs, 
"Bone Reinnervation after Fracture: A Study in the Rat," Journal of Bone and Mineral Research, Vol. 16, No. 8, 2001, pp. 1505-1510. doi:10.1359/jbmr.2001.16.8.1505

[6] J. W. Frymoyer and M. H. Pope, "Fracture Healing in the Sciatically Denervated Rat," The Journal of Trauma, Vol. 17 , No. 5, 1977, pp. 355-361. doi:10.1097/00005373-197705000-00004

[7] M. Hukkanen, Y. T. Konttinen, S. Santavirta, L. Nordsletten, J. E. Madsen, R. Almaas, A. B. Oestreicher, T. Rootwelt and J. M. Polak, "Effect of Sciatic Nerve Section on Neural Ingrowth into the Rat Tibial Fracture Callus," Clinical of Orthopaedics and Related Research. Vol. 311, 1995, pp. 247-257.

[8] J. E. Madsen, M. Hukkanen, A. K. Aune, I. Basran, J. F. Møller, J. M. Polak and L. Nordsletten, "Fracture Healing and Callus Innervation after Peripheral Nerve Resection in Rats," Clinical of Orthopaedics and Related Research, Vol. 351, 1998, pp. 230-240. doi:10.1097/00003086-199806000-00028
[9] R. A. Hayda, C. T. Brighton and J. L. Esterhai, "Pathophysiology of Delayed Healing," Clinical of Orthopaedics and Related Research, Vol. 355, 1998, pp. 31-40. doi:10.1097/00003086-199810001-00005

[10] N. Yüce, B. Gülman and F. Karagöz, "Periferik Sinir Lezyon-Larının Kırık Iyileşmesi Üzerine Etkisi (in Turkish)," Turkish Journal of Medical Research, Vol. 6, No. 5, 1988, pp. 391-396.

[11] H. Aro, "Effect of Nerve Injury on Fracture Healing: Callus Formation Studied in the Rat," Acta Orthopaedica Scandinavica, Vol. 56, No. 3, 1985, pp. 233-237. doi:10.3109/17453678508993002

[12] M. Gajda, J. A. Litwin, T. Cichocki, J. P. Timmermans and D. Adriaensen, "Development of Sensory Innervation in Rat Tibia: Co-Localization of CGRP and Substance P with Growth-Associated Protein 43 (GAP-43)," Journal of Anatomy, Vol. 207, No. 2, 2005, pp. 135-144. doi:10.1111/j.1469-7580.2005.00434.x 